\title{
Photon transfer in ultrastrongly coupled three-cavity arrays
}

\author{
S. Felicetti, ${ }^{1,}{ }^{*}$ G. Romero, ${ }^{1}$ D. Rossini, ${ }^{2}$ R. Fazio, ${ }^{2,3}$ and E. Solano ${ }^{1,4}$ \\ ${ }^{1}$ Department of Physical Chemistry, University of the Basque Country UPV/EHU, Apartado 644, 48080 Bilbao, Spain \\ ${ }^{2}$ NEST, Scuola Normale Superiore and Istituto di Nanoscienze - CNR, Pisa, Italy \\ ${ }^{3}$ Centre for Quantum Technologies, National University of Singapore, Republic of Singapore \\ ${ }^{4}$ IKERBASQUE, Basque Foundation for Science, Alameda Urquijo 36, 48011 Bilbao, Spain
}

(Received 2 May 2013; published 31 January 2014)

\begin{abstract}
We study the photon transfer along a linear array of three coupled cavities where the central one contains an interacting two-level system in the strong- and ultrastrong-coupling regimes. We find that an inhomogeneously coupled array forbids a complete single-photon transfer between the external cavities when the central one performs a Jaynes-Cummings dynamics. This is not the case in the ultrastrong-coupling regime, where the system exhibits singularities in the photon transfer time as a function of the cavity-qubit coupling strength. Our model can be implemented within the state-of-the-art circuit quantum electrodynamics technology and it represents a building block for studying photon state transfer through scalable cavity arrays.
\end{abstract}

DOI: 10.1103/PhysRevA.89.013853

PACS number(s): 42.50.Pq, 03.67.Hk, 42.50.Ct, 85.25.Cp

\section{INTRODUCTION}

Light-matter interaction controls some of the most fundamental processes in nature [1]. Its study has allowed the development of impressive architectures where fundamentals of quantum mechanics can be tested [2]. The high level of control achieved on these setups [3-5] motivated an increasing interest in the study of strongly correlated systems and collective phenomena. In particular, photon lattice models involving coupled cavity arrays have been devised in such a way that each cavity interacts with a single two-level system (qubit), thus realizing the Jaynes-Cummings-Hubbard model [6-8]. The possibility of implementing different geometries enables one to engineer quantum networks for distributed quantum information processing [9]. These lattice models have proved useful to describe the scattering of a single-photon interacting with a qubit in a one-dimensional waveguide [10]. A similar approach can be used to consider homogeneously coupled cavity arrays [11], where the central cavity-qubit interaction is in the strong-coupling (SC) regime. However, when the rotating-wave approximation (RWA) ceases to be valid, the single-photon scattering properties have been only recently studied [12]. Notice that circuit QED technologies [13-15], where photon lattice models have been proposed [16-18], include disorder in the cavity coupling distribution due to imperfections. In addition, circuit QED has allowed the advent of the ultrastrong-coupling (USC) regime of lightmatter interaction [19-21], described by the quantum Rabi model [22]. The latter displayed important consequences in strongly correlated systems, where a $\mathbb{Z}_{2}$ parity-breaking quantum phase transition was predicted [23].

Here, we consider the problem of photon transfer in a linear array of three coupled cavities, where a two-level system interacts at the central site in the SC and USC regimes (see Fig. 1). This configuration can be thought of as a microwave analog [24] of the superconducting Josephson interferometer. We also include disorder in the cavity-cavity coupling, thus mimicking experimental imperfections. Under these conditions, we are able to unveil the following features: (i) in the SC

\footnotetext{
*felicetti.simone@gmail.com
}

regime, and for finite values of the hopping amplitudes and of the cavity-qubit coupling, a single excitation initially localized in the leftmost cavity is strictly forbidden to fully populate the rightmost cavity, similar to the delocalization-localization phenomenon [25]; (ii) in the USC regime, the above restriction does not hold any more, and a single excitation can fully populate the rightmost cavity for almost arbitrary Hamiltonian parameters. Furthermore, the tunneling rate of the single excitation becomes negligible for a critical value of the cavityqubit coupling strength, and it also allows an operational way of defining the SC-USC crossover. Our scheme represents a feasible building block $[16-18,26]$ to study photon excitation and state transfer towards scalable cavity arrays.

The present paper is organized as follows. In Sec. II, we introduce the model and we provide a description of the dynamical processes we consider. In Sec. III, we analyze the single-photon transfer in the SC regime. In Sec. IV, we begin with a discussion on the system dynamics in the case in which the RWA breaks down, before moving on to a comparison of the photon transfer behavior between the SC and USC regimes. Further details on the transfer of a more general state (such as a qubit and a coherent state) are provided in the Appendix. In Sec. V, we report on a particular regime in which the qubit frequency vanishes. Finally, in Sec. VI, we summarize and discuss our results.

\section{MODEL}

Our model consists of an array of three single-mode cavities, where the central site interacts with a two-level system in the SC or in the USC regime. A schematic representation is shown in Fig. 1 where each cavity is linked with its neighbor through a hopping interaction that, in general, is not weak enough to consider the RWA. The corresponding Hamiltonian reads

$$
\begin{aligned}
H= & \sum_{\ell=1}^{3} \omega_{\ell} a_{\ell}^{\dagger} a_{\ell}+\frac{\omega_{q}}{2} \sigma_{z}+g \sigma_{x}\left(a_{2}^{\dagger}+a_{2}\right) \\
& -\sum_{\ell=1}^{2} J_{\ell}\left(a_{\ell}^{\dagger} a_{\ell+1}+a_{\ell}^{\dagger} a_{\ell+1}^{\dagger}+\text { H.c. }\right),
\end{aligned}
$$




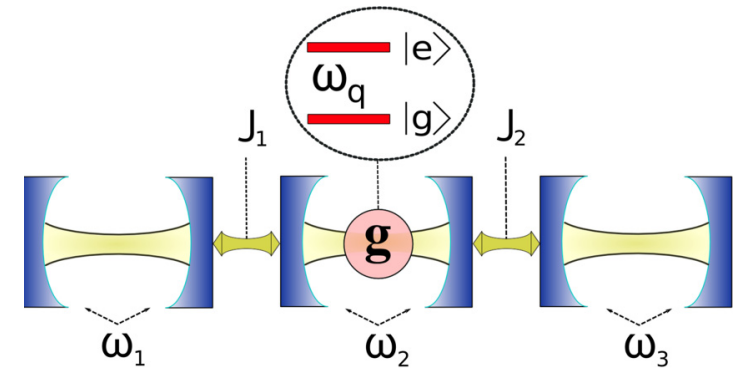

FIG. 1. (Color online) Linear chain of three microwave cavities. The central cavity is coupled to a two-level quantum system in the strong-coupling or ultrastrong-coupling regime. The side cavities are linked to the central one through hopping interaction that can also be strong enough to invalidate the RWA.

where $a_{\ell}\left(a_{\ell}^{\dagger}\right)$ is the annihilation (creation) operator for photons on the $\ell$ th cavity $(\ell=1,2,3), \omega_{\ell}$ being the characteristic frequencies and $J_{\ell}$ being the nearest-neighbor hopping amplitudes. The qubit of frequency $\omega_{q}$ is located inside the central cavity and is described by the Pauli matrices $\sigma_{\alpha}(\alpha=$ $x, y, z$ ), while $g$ denotes the cavity-qubit coupling strength. The complexity of the Hamiltonian in Eq. (1) is associated with the appearance of counter-rotating terms in the cavity-qubit and cavity-cavity interaction. Hereafter, we consider identical cavities $\left(\omega_{\ell}=\omega\right)$ and resonant qubit $\left(\omega_{q}=\omega\right)$. We stress that in all simulations we set the energy scales in units of the resonator frequency $\omega$.

The system is initialized in the state $\left|\psi_{0}\right\rangle=|100\rangle \otimes|g\rangle$, corresponding to having a single photon in the leftmost cavity, zero in the others, and the qubit in its ground state. We address the dynamics dictated by the Hamiltonian (1), and study the single-excitation transfer along our three-cavity array. Depending on the ratio $g / \omega$, two regimes can be identified: the SC regime for $g / \omega \lesssim 0.1$, and the USC regime for $0.1 \lesssim g / \omega \lesssim 1$.

\section{SINGLE-PHOTON TRANSFER IN SC REGIME}

When $g / \omega \ll 1$, the RWA provides a faithful description of the cavity-qubit dynamics, so that we can neglect the counterrotating terms in Eq. (1): $\sigma_{x}\left(a_{2}^{\dagger}+a_{2}\right) \rightarrow\left(\sigma_{+} a_{2}+\sigma_{-} a_{2}^{\dagger}\right)$. In the regime where hoppings $J_{l} / \omega \ll 1$, the cavity-cavity RWA also holds, and the $U(1)$ symmetry provides the conservation of the total number of excitations. In this case, the time evolution of the system will necessarily lead to a state of the form $|\psi(t)\rangle=$ $\alpha|000\rangle \otimes|e\rangle+(\beta|100\rangle+\gamma|010\rangle+\delta|001\rangle) \otimes|g\rangle$. A full analytical solution can be found in the interaction picture and directly solving the Schrödinger equation. At resonance, where qubit and resonator frequencies coincide $\left(\omega_{q}=\omega\right)$, we can derive explicitly the probability amplitude for finding a photon in the rightmost cavity: $\delta(t)=T[\cos (\lambda t)-1] / 2$, where $\lambda=$ $\sqrt{g^{2}+J_{1}^{2}+J_{2}^{2}}$ and the amplitude reads

$$
T=\frac{2 J_{1} J_{2}}{g^{2}+J_{1}^{2}+J_{2}^{2}}
$$

The above result unveils a competition between cavitycavity and cavity-qubit interaction. Equation (2) indeed shows
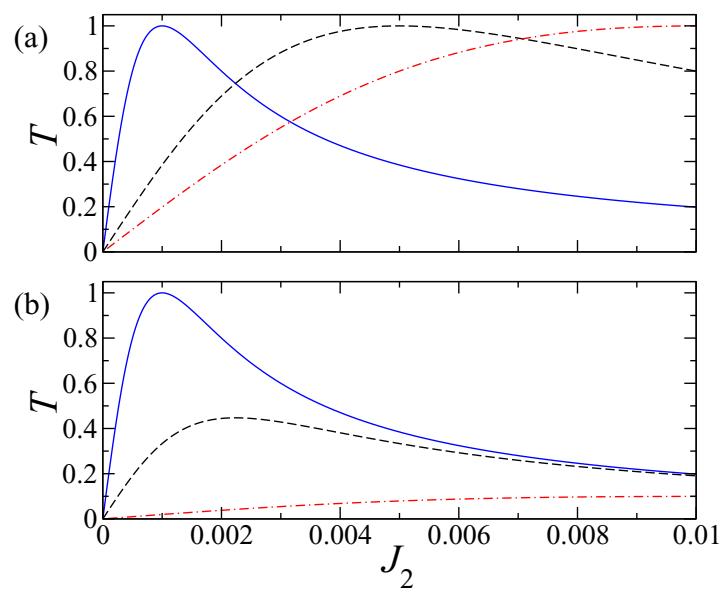

FIG. 2. (Color online) Oscillation amplitude $T$ defined in Eq. (2) as a function of the hopping parameter $J_{2}$, and for different sets of Hamiltonian parameters. In panel (a), for a fixed value of the cavityqubit coupling $g=0$, we display data for $J_{1}=0.001$ (continuous blue line), $J_{1}=0.005$ (dashed black line), and $J_{1}=0.01$ (dot-dashed red line). In panel (b), for a fixed value of $J_{1}=0.001$, we consider $g=0$ (continuous blue line), $g=0.002$ (dashed black line), and $g=0.01$ (dot-dashed red line). Here and in the following figures, we express all the couplings and hoppings in units of $\omega$, while times are denoted in units of $\omega^{-1}$. We also set $\hbar=1$.

that, introducing disorder in the cavity-cavity couplings $\left(J_{1} \neq J_{2}\right)$, the single-photon transfer has a counterintuitive dependence on the hopping terms. In particular, for given parameters $g, \omega$, and $J_{1}$, the excitation transfer to the rightmost cavity exhibits a nonmonotonic behavior with increasing $J_{2}$ and is maximum for $J_{1}=J_{2}$ [see Fig. 2(a)]. Note that only in the homogeneous case $\left(J_{1}=J_{2}\right)$ and for a negligible cavity-qubit coupling $g$ is it possible to have photon transfer with unit probability. On the other hand, for any finite value of $g$, the latter is strictly forbidden. We also notice that, in Eq. (2) and for fixed $J_{1}$, increasing $g$ decreases the amplitude $T$ [see Fig. 2(b)]. This behavior has a simple explanation: when a single excitation tries to move from the left to the central cavity, it is scattered back by the cavity-qubit system without being fully absorbed. In fact, the probability for exciting the qubit $|\alpha|^{2}$ is inversely proportional to the square of the coupling strength.

\section{SINGLE-PHOTON TRANSFER IN THE USC REGIME}

If the coupling strength $g$ and the resonator frequency $\omega$ satisfy $0.1 \lesssim g / \omega \lesssim 1$, the system enters the USC regime. In this case, photons are spontaneously generated from the vacuum such that the total number of excitations grows with the ratio $g / \omega$, enlarging unavoidably the associated Hilbert space [27]. To observe an appreciable excitation transfer, the photon hopping strength must be of the same order of $g$; thus we consider values up to $J_{\ell} \sim 0.1$. In this regime, for the sake of consistency, the counter-rotating terms of the cavity-cavity interaction have been taken into account, although they do not change qualitatively the system dynamics. In order to provide a reliable system real-time dynamics, we performed a fourthorder-Trotter expansion of the evolution operator $[28,29]$. To 

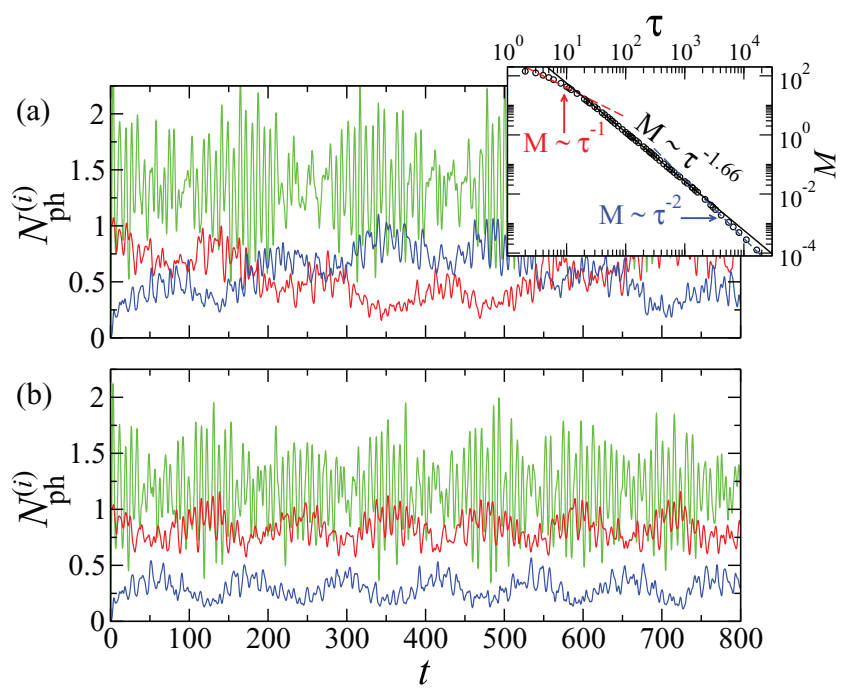

FIG. 3. (Color online) Average photon number in each cavity $N_{\mathrm{ph}}^{(i)}(t)$ at resonance condition $\left(\omega_{q}=\omega\right)$, and for homogeneous cavity-cavity couplings $\left(J_{1}=J_{2}=0.1\right)$. The red line stands for the leftmost cavity and the blue line for the rightmost cavity, while the green line stands for the central cavity. The main panels refer to different cavity-qubit couplings: $g=0.9$ (a); $g=0.85$ (b). The inset shows the box counting analysis for $N_{\mathrm{ph}}^{(1)}(t)(g=0.9)$, and displays $M$ as a function of $\tau$ (see the main text and Ref. [31]). A power-law fit of the intermediate region gives a fractal dimension $\mathscr{D} \approx 1$.66. Time is expressed in units of $\omega^{-1}$.

extend this kind of analysis to a higher number of cavities, one should rely on more sophisticated numerical techniques, such as the time-evolving block-decimation scheme for a onedimensional array of cavities.

\section{A. System dynamics}

Figure 3 shows the time evolution of the average photon number in each cavity, $N_{\mathrm{ph}}^{(i)}(t)=\left\langle\psi(t)\left|a_{i}^{\dagger} a_{i}\right| \psi(t)\right\rangle$, starting from the state $\left|\psi_{0}\right\rangle=|100\rangle \otimes|g\rangle$ that evolves according to Hamiltonian (1). At first glance, one recognizes a highly irregular behavior of $N_{\mathrm{ph}}^{(i)}(t)$, which arises from the counterrotating terms in the cavity-qubit interaction. Remarkably, this is developed by the unitary evolution of the system itself, and it is not due to the limited time resolution of our simulations. In order to quantify this behavior, we analyze the fractal dimension of $N_{\mathrm{ph}}^{(1)}(t)$ by using the modified box counting algorithm [30]. This consists in dividing the total time interval in segments of size $\tau$, and then covering the data with a set of rectangular boxes of size $\tau \times \Delta_{i}\left(\Delta_{i}\right.$ is the largest excursion of the curve in the $i$ th region $\tau$ ). One then computes the average excursion $M(\tau)=\sum_{i} \Delta_{i} / \tau$. The dimension $\mathscr{D}$ of the curve is defined by $\mathscr{D}=-\log _{\tau} M(\tau)$. One finds $\mathscr{D}=1$ for a straight line, and $\mathscr{D}=2$ for a periodic curve. Indeed, for times much larger than the period, a periodic curve uniformly covers a rectangular region. Any value of $\mathscr{D}$ between these integer values entails the fractality of the curve [31]. In the case of Fig. 3(a), we obtained $\mathscr{D} \approx 1.66$ (inset to the figure). Furthermore, increasing $g$, we found a fractal dimension which rapidly decreases from $\mathscr{D} \sim 2$ (quasiperiodic curve) down to noninteger values close to $\mathscr{D} \sim 1.5$ for large cavity-qubit couplings.

The fractal time dependence emerging in the system is clearly due to the presence of counter-rotating terms. Here we point out that a similar analysis, performed after removing the two side cavities from the model, systematically produced integer values of $\mathscr{D}$. This means that the Rabi model alone is not sufficient to generate a fractal signal. In order to have a sufficient number of incommensurate frequencies generating a fractal behavior, the Rabi model has to be combined with some nontrivial interaction with other bosonic modes.

\section{B. Photon transfer}

Before discussing the transfer properties, it is appropriate to observe that, even though in the USC regime photons are spontaneously generated in the central site, for the coupling values considered here and if the system is initialized in the vacuum state, the average photon number in the side cavities remains much smaller than 0.1 during the whole evolution. This guarantees that the signal tunneled through the central site can be clearly distinguished from excitations generated by the presence of counter-rotating terms. Furthermore, in the case of homogeneous cavity-cavity couplings, given the symmetry of the system Hamiltonian (1), the two side cavities have identical behavior and differences between them can arise only from asymmetrical initial states.

Two fundamental differences with respect to the SC regime can be found in the excitation transfer analysis. First, in the USC regime a single-photon excitation can be completely transferred from the leftmost to the rightmost cavity, also for a finite value of the coupling strength $g$ [see, e.g., Fig. 3(a)]. This is the typical situation for almost arbitrary Hamiltonian parameters in the USC regime, a disparity that can be explained as follows. The initial state $\left|\psi_{0}\right\rangle$ has a finite overlap with the state $|E\rangle=(g|100\rangle \otimes|g\rangle+J|000\rangle \otimes|e\rangle) / \sqrt{g^{2}+J^{2}}$. In the $\mathrm{SC}$ regime, $|E\rangle$ is a Hamiltonian eigenstate, and its overlap with the evolved system state $|\psi(t)\rangle$ is conserved during the time evolution. Consequently, the state $|001\rangle \otimes|g\rangle$ is not accessible (as far as $g \neq 0$ ). On the other hand, in the USC regime the state $|E\rangle$ is no longer an eigenstate of the Hamiltonian. Hence the previous limitation does not hold anymore, and complete transfer to the rightmost cavity is generally allowed. In the USC regime, the qubit and the field in the central cavity cannot be considered as separated entities, and the Jaynes-Cummings doublets are not the correct eigenstates to describe the system dynamics. In fact, the system eigenstates are defined in two infinite-dimensional Hilbert spaces that have a defined parity $p= \pm 1$, according to the $\mathbb{Z}_{2}$ symmetry [22].

The second feature to be highlighted is that the photon transfer is strongly inhibited for a specific value of the cavity-qubit coupling strength, as displayed in Fig. 3(b). In particular, in Fig. 4 we analyzed the population inversion time $T_{\text {inv }}$, defined as the time in which the average photon number becomes bigger in the rightmost cavity than in the leftmost cavity, as a function of the coupling strength $g$, and for different values of the cavity-cavity coupling strength. We found that there exists a critical value $g_{c}$, dependent on the system parameters, for which the time needed to observe single-photon transfer 


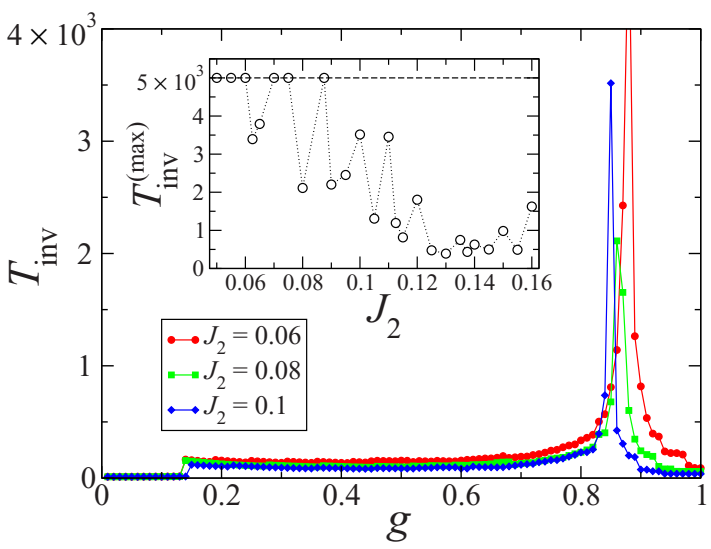

FIG. 4. (Color online) Population inversion time $T_{\text {inv }}$ as a function of the cavity-qubit coupling constant, for $J_{1}=0.1$, defined as the time in which $N_{\mathrm{ph}}^{(3)}(t)$ becomes bigger than $N_{\mathrm{ph}}^{(1)}(t)$. We note the SC-USC transition for $g \approx 0.14$, and the inhibition of state transfer around a critical value $g_{c}$, which depends on the hopping term $J_{2}$ according to $g_{c} \approx 0.94-0.97 \times J_{2}$. The couplings $g$ and $J_{1,2}$ are expressed in units of $\omega$, as well as $T_{\text {inv }}$ in units of $\omega^{-1}$. The inset displays the maximum value of the population inversion time $T_{\mathrm{inv}}^{(\max )}$ that is reached at $g_{c}$, as a function of $J_{2}$ [29].

dramatically increases. For the considered parameter ranges, we find a critical coupling $0.78 \lesssim g_{c} \lesssim 0.88$ (in units of $\omega$ ). Fixing $J_{1}=0.1$, we found $g_{c} \approx 0.94-0.97 \times J_{2}$, while the population inversion time $T_{\text {inv }}^{(\max )}$ in correspondence to such a value exhibits a quite irregular pattern of oscillations with $J_{2}$ (inset to Fig. 4). This phenomenon is specific to the USC dynamics and occurs in the higher-coupling region of the Rabi model $(g / \omega \gtrsim 0.4)$ [32], a zone where the photon production exceeds the RWA predictions, and an analytical treatment becomes difficult despite the integrability of the model [22].

We highlight the sudden increase of $T_{\text {inv }}$ occurring in the lower-coupling region of the Rabi model, which is zoomed-in in Fig. 5. This abrupt behavior is due to the SC-USC regime crossover. If the RWA holds, the population inversion time

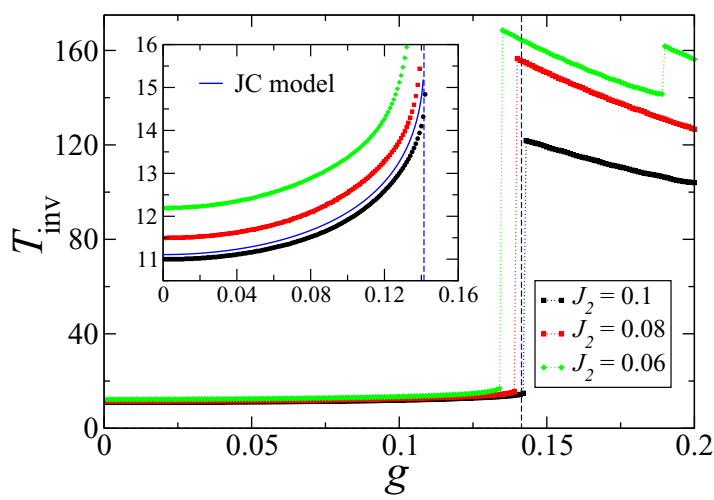

FIG. 5. (Color online) Population inversion time $T_{\text {inv }}$ (in units of $\omega^{-1}$ ) as a function of the cavity-qubit coupling constant $g$, and for fixed value $J_{1}=0.1$. The blue and the black lines correspond to the homogeneous case. The blue line corresponds to the analytical solution when the RWA holds for cavity-cavity and cavity-qubit interaction. The black, red, and green lines are obtained through numerical simulations of the full model ruled by the Hamiltonian (1). is given by $T_{\text {inv }}=\arccos \left[1-\lambda^{2} /\left(J_{1}^{2}+J_{2}^{2}\right)\right] / \lambda$, where $\lambda$ has been defined previously. For $g>\sqrt{J_{1}^{2}+J_{2}^{2}}$, the population inversion never occurs. Contrariwise, when the counter-rotating terms are taken into account, we observe population inversion at finite time also for a larger value of the coupling $g$. Notice that the location of the discontinuities appearing in Fig. 5 is related to our definition for the inversion time $T_{\text {inv }}$. For example, a redefinition of $T_{\mathrm{inv}}$ as the time in which $N_{\mathrm{ph}}^{(3)} / 3$ becomes bigger than $2 N_{\mathrm{ph}}^{(1)} / 3$ would quantitatively change the value of $g$ for which the inversion time experiences a discontinuity. However, the emerging physics would not qualitatively change. Here we use the most natural definition of $T_{\mathrm{inv}}$, in analogy with the concept of population inversion in statistical mechanics. The sudden increase of the population inversion time is due to the fact that, in the USC regime, the accessible part of the Hilbert space is unbounded, because of the $U(1)$ symmetry breaking down to a $\mathbb{Z}_{2}$ symmetry. Hence the system can explore a much bigger number of states: complete transfer is allowed, but the probability of its occurrence is smaller. For our three-cavity array, the parameter $g_{t}=g / \sqrt{J_{1}^{2}+J_{2}^{2}}$ provides us with an operational definition for the SC-USC transition of the cavity-qubit interaction. For small values of $g_{t}$, the RWA describes correctly the transfer dynamics. When $g_{t}>1$, the full model behavior differs quantitatively and qualitatively from the JC model predictions. We observe also that varying $J_{2}$ with respect to $J_{1}$ results in a longer population inversion time: in both regimes, inhomogeneity in the hopping terms hinders the excitation transfer.

We have also analyzed the transfer of coherent states, $\left|\phi_{0}\right\rangle=$ $|\alpha\rangle$, and of arbitrary linear superposition states $\left|\phi_{0}\right\rangle=p|0\rangle+$ $e^{i \theta} \sqrt{1-p^{2}}|1\rangle$, with $p$ randomly chosen in the interval $[0,1]$. We numerically simulated the system evolution, setting $\left|\psi_{0}\right\rangle=$ $\left|\phi_{0}\right\rangle|0\rangle|0\rangle|g\rangle$ as initial state, and we recorded the behavior of the transfer fidelity, defined as $F=\operatorname{Tr}\left[\rho_{0} \rho(t)\right]$, where $\rho_{0}=$ $\left|\phi_{0}\right\rangle\left\langle\phi_{0}\right|$ and $\rho(t)$ is the state of the rightmost cavity at time $t$. The results we found are consistent with those relative to the case of single-excitation transfer. The interplay between the hopping constant $J$ and the cavity-qubit coupling strength $g$ rules the state transfer dynamics: with increasing $J$ the transfer is more likely to happen, while increasing $g$ results in smaller values of the transfer fidelity. Specifically, in the SC regime, as far as $g \neq 0$, it is impossible to observe complete state transfer, i.e., $F<1$ at any time. This is not the case in the USC regime, where the transfer fidelity, in a linear superposition or a coherent state, can be close to unity; also when $g$ and $J$ are of the same order. These results show the peculiar features of state transfer physics beyond the RWA, and may pave the way for developing a general theory in the presence of USC regimes. Further details on state transfer features can be found in the Appendix.

\section{DEGENERATE QUBIT CASE}

In the case in which the qubit frequency vanishes, a closed analytical solution of the system dynamics is available if we consider the RWA in the cavity-cavity interaction. This model can be simulated with current technology by means 
of a coupled cavity-qubit system in the SC regime, and the application of a strong classical driving to the qubit $[33,34]$. It can be shown that the dynamics of such a system will be ruled by the effective Hamiltonian

$$
H_{\mathrm{eff}}=\omega \sum_{\ell=1}^{3} a_{\ell}^{\dagger} a_{\ell}+g \sigma_{x}\left(a_{2}^{\dagger}+a_{2}\right)-\sum_{\ell=1}^{2} J_{\ell}\left(a_{\ell}^{\dagger} a_{\ell+1}+\text { H.c. }\right)
$$

which can be obtained setting $\omega_{q}=0$ in Eq. (1), and performing the RWA on the cavity-cavity interaction. In this case, the excitation transfer exhibits a smooth periodic behavior in time, which is independent of the cavity-qubit coupling strength, and allows a complete transfer at regular times. In fact, the time evolution of the difference between the average photon number in the leftmost and rightmost cavities, starting from the initial state $\left|\psi_{0}\right\rangle=|100\rangle \otimes|g\rangle$ in the homogeneous case, reads

$$
\Delta N_{\mathrm{ph}}(t)=N_{\mathrm{ph}}^{(1)}(t)-N_{\mathrm{ph}}^{(3)}(t)=\cos (\sqrt{2} J t) .
$$

This result holds both in the SC and in the USC regime; hence the counter-rotating terms do not modify the excitation transfer properties of the system. We point out that, when $g / \omega \gtrsim 0.1$, spontaneous photon generation occurs: $\left\langle a_{1}^{\dagger} a_{1}\right\rangle$ and $\left\langle a_{3}^{\dagger} a_{3}\right\rangle$ keep a chaotic time dependence, despite their difference follows a smooth and regular behavior.

\section{CONCLUSIONS AND OUTLOOK}

We studied excitation transfer in an array of three coupled cavities, where a two-level system interacts with the central one, focusing on the comparison between strong- and ultrastrong-coupling regimes of light-matter interaction. In the $\mathrm{SC}$ regime, the cavity-qubit interaction $g$ and the cavity-cavity inhomogeneities $J_{1} \neq J_{2}$ constrain the excitation transfer, thus inhibiting complete population tunneling. On the contrary, in the USC regime a much richer scenario appears. A complete photon transfer is generally allowed, even for finite values of the cavity-qubit coupling strength and for inhomogeneous hoppings, although there exists a specific regime for which the tunneling rate becomes negligible. The complexity of the USC dynamics, generated by counter-rotating terms, manifests itself in the highly irregular time pattern of the observables, exhibiting a fractal behavior. Nonetheless, the physics beyond the RWA plays an important role for the enhancement of quantum state transfer. Finally, in the degenerate qubit case, the excitation transfer is regular and its period does not depend on the cavity-qubit coupling strength.

The proposed scheme can be implemented with state-ofthe-art superconducting circuit technology or, alternatively, its quantum simulation may be considered. Furthermore, it can be used as a building block for realizing controllable quantum simulation of large lattices involving the quantum Rabi model in all coupling regimes.

We also remark that studying the photon transport mechanism in coupled cavities, as for example our three-cavity setup, in the presence of losses could be also relevant for the understanding of noise-assisted transport in quantum networks $[35,36]$.

\section{ACKNOWLEDGMENTS}

We acknowledge fruitful discussions with F. Ciccarello. This work was supported by the Basque Government IT47210, Spanish MINECO FIS2012-36673-C03-02, UPV/EHU UFI 11/55, PROMISCE, CCQED, SCALEQIT, SOLID, and SIQS European projects.

\section{APPENDIX: FURTHER DETAILS ON THE STATE TRANSFER}

\section{State transfer: Linear superposition}

Here we consider the state transfer of a Fock state linear superposition $|0\rangle$ and $|1\rangle$, containing zero and one excitations of the cavity mode, respectively. For simplicity, we have considered homogeneous cavity-cavity couplings, $J_{1}=J_{2}$, and resonance condition, $\omega_{q}=\omega$. The system is initialized in state $\left|\psi_{0}\right\rangle=\left|\phi_{0}\right\rangle|0\rangle|0\rangle|g\rangle$, where $\left|\phi_{0}\right\rangle=p|0\rangle+$ $e^{i \theta} \sqrt{1-p^{2}}|1\rangle$, with $p$ randomly chosen between 0 and 1 . We define the state transfer fidelity as $F=\operatorname{Tr}\left[\rho_{0} \rho(t)\right]$, that is a measure of the overlap of the rightmost cavity state $\rho(t)$ with the leftmost cavity initial state $\rho_{0}=\left|\phi_{0}\right\rangle\left\langle\phi_{0}\right|$.

The results for different system parameters are shown in Fig. 6. On one hand, in Fig. 6(a) we have chosen a cavity-qubit coupling in the SC regime, $g / \omega=0.01$, and two values for the cavity-cavity coupling, $J / \omega=0.01,0.1$. First, when both quantities are in the SC regime, the cavity-qubit interaction sets an upper bound for the maximum of the state transfer fidelity (black line). Second, if the cavity-cavity coupling is in the USC regime, $J / \omega=0.1$, then almost complete state transfer occurs (red line). On the other hand, in Fig. 6(b), we plot the fidelity $F$ for a cavity-qubit coupling in the USC regime, $g / \omega=0.2$ and $J / \omega=0.01,0.1$ for the cavity-cavity coupling. When $g$ is one order of magnitude larger than $J$ (black line), the transfer is completely inhibited. Contrariwise, there is an enhancement
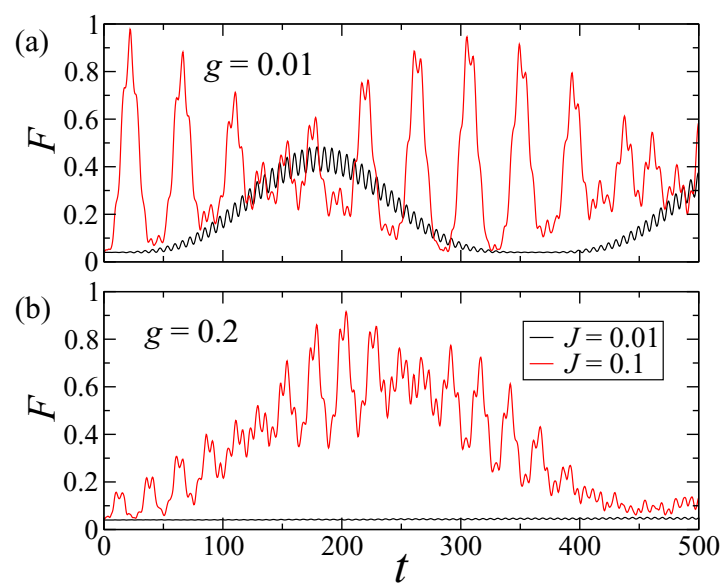

FIG. 6. (Color online) (a) State transfer fidelity $F=\operatorname{Tr}\left[\rho_{0} \rho(t)\right]$ over time for different system parameters. We define $\left|\phi_{0}\right\rangle=p|0\rangle+$ $e^{i \theta} \sqrt{1-p^{2}}|1\rangle$, with $p=0.2$ and $\theta=0.63$. In the SC regime, that is when $g=J=0.01$ (black line), the state transfer fidelity is bounded: it cannot reach 1 as far as $g \neq 0$. (b) When counter-rotating terms are involved in the dynamics $(J \gtrsim 0.1$ and/or $g \gtrsim 0.1)$, the fidelity can be close to 1 even when $J<g$ (red line). Time is expressed in units of $\omega^{-1}$. 

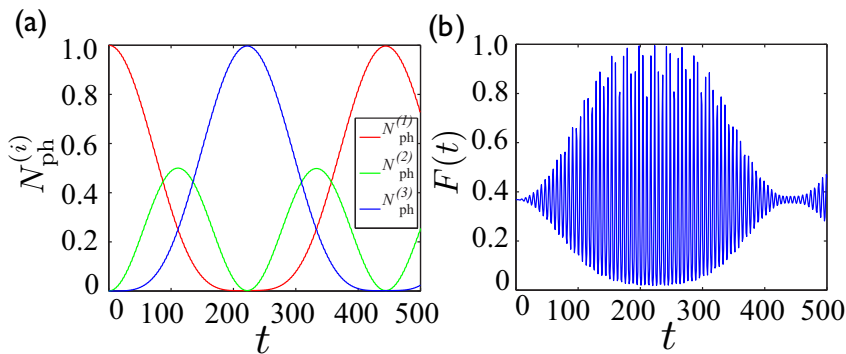

FIG. 7. (Color online) Transfer of a coherent state of amplitude $\alpha=1$ along a three-cavity array $(g=0)$. (a) Average photon number for the leftmost (red), central (green), and rightmost (blue) cavities. (b) State transfer fidelity over time. In the case of qubit absence, the coherent state is fully transferred. Observe that the fidelity at time zero has a finite value $F \approx 0.38$, which corresponds to the overlap between the vacuum state and the considered coherent state. Time is expressed in units of $\omega^{-1}$.

in the state transfer when the cavity-cavity coupling is in the USC regime (red line).

\section{State transfer: Coherent state}

In this section, we report the transfer properties of coherent states. We numerically simulated the system dynamics starting from the initial state $\left|\psi_{0}\right\rangle=\left|\phi_{0}\right\rangle|0\rangle|0\rangle|g\rangle$, where $\left|\phi_{0}\right\rangle=|\alpha\rangle$ is a coherent state of amplitude $|\alpha|$. For the sake of clarity, we first show how a coherent state would transfer along a three-cavity array in the absence of the qubit interaction. This corresponds to setting $g=0$ in our model. Such dynamics is shown in Fig. 7 for a coherent state with $\alpha=1$. In Fig. 7(a), we show the average photon number for the three cavities, while Fig. 7(b) contains the state transfer fidelity. These figures show that the coherent state crosses the central cavity and it is recomposed in the rightmost one. The fast-oscillating behavior of the fidelity is due to relative phase rotation of different coherent state components. We observe also that the fidelity oscillates around a value slightly smaller than $F=0.4$ due (a)

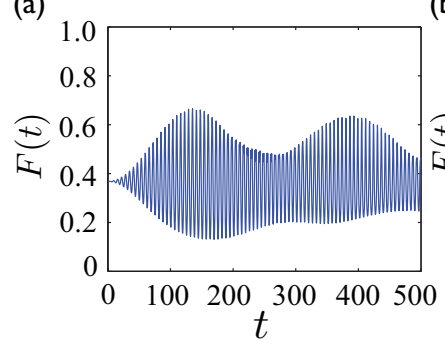

(b)

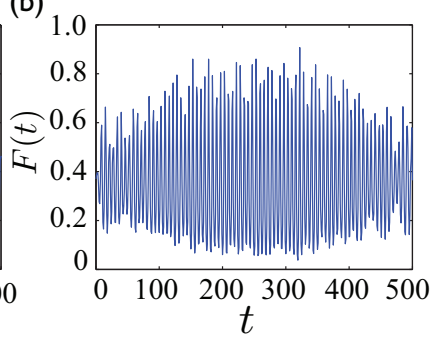

FIG. 8. (Color online) (a) State transfer fidelity over evolution time (in units of $\omega^{-1}$ ), for a coherent state of amplitude $\alpha=1$. The system parameters are given by $g=0.02$ and $J=0.01$ (SC regime). (b) State transfer fidelity over evolution time, for a coherent state of amplitude $\alpha=1$. The system parameters are given by $g=0.2$ and $J=0.1$ (USC regime). The maximum value that the fidelity can reach is not directly bounded by the cavity-qubit interaction.

to the finite overlap between the coherent state $|\alpha\rangle$ and the vacuum state $|0\rangle$.

Let us now consider our full model, composed of three cavities and a qubit interacting with the central one. The transfer of coherent states follows the same general rules reported for the case of the linear superposition state, even if the time-dependent fidelity has a fast-oscillating behavior. In the SC regime, the cavity-qubit interaction limits the maximum value that the fidelity can reach. Figure 8(a) shows the time evolution of the state transfer fidelity in the case in which $J=0.01$ and $g=0.01$, where complete transfer is not allowed. In contrast, in the USC regime, high values of the fidelity can be reached also when $g$ is larger than $J$. Figure 8(b) shows the coherent state transfer dynamics, for a case in which both the cavity-cavity and the cavity-qubit interactions are in the USC regime. The plot shows the fidelity $F$ over evolution time for a coherent state of amplitude $|\alpha|=1$. In this case, the fidelity can reach $F=0.9$, also if $g=2 \mathrm{~J}$.

The cases considered in this Appendix (linear superpositions and coherent states) display the main features of the state transfer when considering the physics beyond the RWA.

[1] C. Cohen-Tannoudji, J. Dupont-Roc, and G. Grynberg, Atom-Photon Interactions: Basic Processes and Applications (WILEY-VCH Verlag GmbH \& Co. KGaA, Weinbeim, 2004).

[2] T. D. Ladd, F. Jelezko, R. Laflamme, Y. Nakamura, C. Monroe, and J. L. O'Brien, Nature (London) 464, 45 (2010).

[3] S. Haroche and J.-M. Raymond, Exploring the Quantum (Oxford University Press, Inc., New York, 2006); H. Walther, B. T. H. Varcoe, B. G. Englert, and T. Becka, Rep. Prog. Phys. 69, 1325 (2006).

[4] I. Bloch, Nat. Phys. 1, 23 (2005).

[5] R. Blatt and C. F. Roos, Nat. Phys. 8, 277 (2012).

[6] M. J. Hartmann, F. G. S. L. Brandão, and M. B. Plenio, Nat. Phys. 2, 849 (2006).

[7] A. D. Greentree, C. Tahan, J. H. Cole, and L. C. L. Hollenberg, Nat. Phys. 2, 856 (2006).

[8] D. G. Angelakis, M. F. Santos, and S. Bose, Phys. Rev. A 76, 031805(R) (2007).
[9] S. Ritter, C. Nölleke, C. Hahn, A. Reiserer, A. Neuzner, M. Uphoff, M. Mücke, E. Figueroa, J. Bochmann, and G. Rempe, Nature (London) 484, 195 (2012).

[10] P. Longo, P. Schmitteckert, and K. Busch, J. Opt. A: Pure Appl. Opt. 11, 114009 (2009); Phys. Rev. Lett. 104, 023602 (2010); Phys. Rev. A 83, 063828 (2011).

[11] L. Zhou, Z. R. Gong, Y.-X. Liu, C. P. Sun, and F. Nori, Phys. Rev. Lett. 101, 100501 (2008).

[12] Z. H. Wang, Y. Li, D. L. Zhou, C. P. Sun, and P. Zhang, Phys. Rev. A 86, 023824 (2012).

[13] A. Blais, R. S. Huang, A. Wallraff, S. M. Girvin, and R. J. Schoelkopf, Phys. Rev. A 69, 062320 (2004).

[14] I. Chiorescu, P. Bertet, K. Semba, Y. Nakamura, C. J. P. M. Harmans, and J. E. Mooij, Nature (London) 431, 159 (2004).

[15] A. Wallraff, D. I. Schuster, A. Blais, L. Frunzio, R.-S. Huang, J. Majer, S. Kumar, S. M. Girvin, and R. J. Schoelkopf, Nature (London) 431, 162 (2004). 
[16] A. A. Houck, H. Türeci, and J. Koch, Nat. Phys. 8, 292 (2012).

[17] M. Leib, F. Deppe, A. Marx, R. Gross, and M. Hartmann, New J. Phys. 14, 075024 (2012).

[18] D. L. Underwood, W. E. Shanks, J. Koch, and A. A. Houck, Phys. Rev. A 86, 023837 (2012).

[19] J. Bourassa, J. M. Gambetta, A. A. Abdumalikov, Jr., O. Astafiev, Y. Nakamura, and A. Blais, Phys. Rev. A 80, 032109 (2009).

[20] T. Niemczyk, F. Deppe, H. Huebl, E. P. Menzel, F. Hocke, M. J. Schwarz, J. J. García-Ripoll, D. Zueco, T. Hümmer, E. Solano, A. Marx, and R. Gross, Nat. Phys. 6, 772 (2010).

[21] P. Forn-Díaz, J. Lisenfeld, D. Marcos, J. J. García-Ripoll, E. Solano, C. J. P. M. Harmans, and J. E. Mooij, Phys. Rev. Lett. 105, 237001 (2010).

[22] D. Braak, Phys. Rev. Lett. 107, 100401 (2011).

[23] M. Schiró, M. Bordyuh, B. Öztop, and H. E. Türeci, Phys. Rev. Lett. 109, 053601 (2012).

[24] D. Gerace, H. E. Türeci, A. Imamoglu, V. Giovannetti, and R. Fazio, Nat. Phys. 5, 281 (2009).

[25] S. Schmidt, D. Gerace, A. A. Houck, G. Blatter, and H. E. Türeci, Phys. Rev. B 82, 100507(R) (2010).

[26] M. Mariantoni, H. Wang, R. C. Bialczak, M. Lenander, E. Lucero, M. Neeley, A. D. O'Connell, D. Sank, M. Weides, J. Wenner, T. Yamamoto, Y. Yin, J. Zhao, J. M. Martinis, and A. N. Cleland, Nat. Phys. 7, 287 (2011).

[27] The counter-rotating terms in the cavity-cavity coupling also induce photon generation from the vacuum. However, for the cases we consider $\left(J_{\ell} \lesssim g\right)$ most of the nonconserving excitation effects come from the cavity-qubit USC regime.
[28] See, e.g., A. T. Sornborger and E. D. Stewart, Phys. Rev. A 60, 1956 (1999).

[29] In order to keep the Hilbert space dimension manageable in our simulations, we cut the maximum number of allowed photons per cavity to a finite amount $N_{\max }=18$. We checked that such cutoff value induces an error that is negligible on the scale of our results, for $g / \omega \lesssim 1$. All Trotter time evolutions have been performed up to a time $t=5000$.

[30] G. De Chiara, D. Rossini, S. Montangero, and R. Fazio, Phys. Rev. A 72, 012323 (2005).

[31] For any curve, there exists a region of box lengths $\tau_{\min }<$ $\tau<\tau_{\max }$, where $M \propto \tau^{\mathscr{D}}$. Outside this region, one either finds $\mathscr{D}=1$ or $\mathscr{D}=2$. The first equality holds for $\tau<\tau_{\min }$, and it is due to the coarse grain artificially introduced by numerical simulations. The second one is obtained for $\tau>\tau_{\max }$ and it is due to the finite length of the analyzed time series. The boundaries $\tau_{\min }, \tau_{\max }$ have to be chosen properly for any time series.

[32] F. A. Wolf, F. Vallone, G. Romero, M. Kollar, E. Solano, and D. Braak, Phys. Rev. A 87, 023835 (2013).

[33] E. Solano, G. S. Agarwal, and H. Walther, Phys. Rev. Lett. 90, 027903 (2003).

[34] D. Ballester, G. Romero, J. J. García-Ripoll, F. Deppe, and E. Solano, Phys. Rev. X 2, 021007 (2012).

[35] M. B. Plenio and S. F. Huelga, New J. Phys. 10, 113019 (2008).

[36] M. Mohseni, P. Rebentrost, S. Lloyd, and A. Aspuru-Guzik, J. Chem. Phys. 129, 174106 (2008). 